\title{
Molecular Dynamic of (4-(4'Octylobiphenyl)carboxylan) 4-(2-methylobuthyl) phenol Liquid Crystal Having Blue Phase Seen via the Dielectric Spectroscopy Method
}

\author{
W. OTOWski* AND G. LEWIŃsKA \\ Institute of Physics, Cracow University of Technology, Kraków, Poland \\ (Received July 29, 2013; in final form September 23, 2013)
}

\begin{abstract}
(4-(4'octylobiphenyl)carboxylan) 4-(2-methylobuthyl) phenol (CE8), the substance showing complex polymorphism, has been investigated using the dielectric spectroscopy method. This substance exhibited polymorphism during heating and cooling: a few smectic phases, a cholesteric phase, and a blue phase as well. Despite the fact that the substance polymorphism depends on the rate of heat flow existing of the blue phase has been confirmed by polarizing microscopic observations as well as dielectric spectroscopy investigations.
\end{abstract}

DOI: $10.12693 /$ APhysPolA.125.1152

PACS: 61.30.Pq, 61.30.Eb, 64.70.M-, 42.70.Df

\section{Introduction}

Liquid crystals are structures built up of anisotropic molecules. However, the open question is how the one anisotropic molecule as a main structural element may set in order, giving a phase or sequence of different phases. Especially the so called blue phase (BP) arrangement is the most interesting property giving right now attempt to new class of LC displays. On the other hand, the interest in the dynamics of the liquid-crystalline systems (including BP phases) on the understanding that one considers the molecular motions, seems to be important to create the entire "image" of LC phases. Therefore the investigation of the substance, having a relatively simple chemical structure and different phase sequence including the blue phase, seems to be interesting.

History of the blue phase is as long as history of liquid crystals. It appears in narrow temperature regions (about $1{ }^{\circ} \mathrm{C}$ ). However in 2005 , researchers from the University of Cambridge reported their discovery of a class of blue-phase liquid crystals [1]. This new liquid crystals remain stable over a wide temperature range (thermodynamically stable for $16^{\circ} \mathrm{C}-60^{\circ} \mathrm{C}$ ). So this system would be used to switch the colour of the reflected light by applying an electric field to the material that this could eventually be used to produce three-colour (red, green, and blue) pixels for full-colour displays [2]. Thus the blue phase becomes the very interesting mesophase for basic investigation as well as for applications. In 2007 Samsung presented the first LCD "Blue Phase" display which does not require anisotropic alignment layers (e.g., rubbed polyimide) and thus simplified the LCD manufacturing process.

The blue phase (correctly speaking: phases) occurs extremely rarely in the temperature range between

*corresponding author; e-mail: w_otowski@wp.pl cholesteric (a chiral nematic) phase and isotropic liquid phase. It is a state with unusual properties. Generally blue phase has a regular three-dimensional cubic structure. The competition between the chiral forces and a "double twist" structure leads to stable network [3]. There are three stable blue phases labelled BPI, BPII and BPIII upon increasing the temperature from the chiral nematic to isotropic phase. The double twist means structure in which the director twists simultaneously about two independent directions. Order parameter exhibits three-dimensional orientational ordering with periods of up to $500 \mathrm{~nm}$. Selective Bragg reflections exhibit in the visible light wavelength range. Blue phases I (BPI) and II (BPII) show cubic symmetry. For BPI it is body center cubic symmetry, elementary cell size changes with temperature. BPII shows simple cubic symmetry and there is no temperature change in elementary cell size. BPIII, called blue fog, is almost amorphic (chiral positional ordering short-range).

Except that the blue phase is a curiosity of nature, it proves to be quite an interesting system for studying the properties of oriented molecule systems.

Taking into account the dielectric spectroscopy we must realise that this technique monitors macroscopic properties of the system. Dielectric spectroscopy measures the collective response of a charge-free system to an applied electric field. The entire spectral density of the molecular motion comes from a bulk liquid crystal. So, in dielectric experiment one observes the motion of a "probe molecule". Because the dielectric "probe molecule" could not be considered as a real liquid crystal mesogen, one should consider an important problem how to transform a "probe" molecule to a real liquid crystal one [4].

Because of chirality of the investigated material, one may expect two collective processes in ferroelectric SmC phase. Besides the molecular processes, one should observe the Goldstone mode in the whole temperature range and the soft mode close to the transition temperature. Both are strong collective modes observed in planar ge- 
ometry. Soft mode (amplitudon mode) is fluctuations in the angle. The Goldstone helix distortion mode (phason mode) is fluctuations in the phase angle (tilt direction). The low-frequency dielectric measurements of CE8 as the functions of both the temperature and a bias electric field has been done by Cava et al. [5]. They measured CE8 liquid crystal in chiral and recemic forms. Important information is that they observed one Cole-Cole process. However, one should keep in mind that ferroelectric liquid crystal under bias voltage presents new structure. Ferroelectric helix is deformed (helix is partially unwind) and molecular organization is disturbed. Anyhow, we must keep in mind that bias voltage do not generate thermodynamic transition.

In this paper we concentrate on molecular description of the reorientation observed in undisturbed regime. To this end we decided to carry out investigation for bulk sample without any bias voltage.

\section{Material}

The substance under study (4-(4'octylobiphenyl)carboxylan) 4-(2-methylobuthyl) phenol liquid crystal was synthesized in the Institute of Chemistry, the Military University of Technology in Warsaw. It was preliminarily studied there and gave reasons to suspect of wide variety mesophases. The compound is chiral, with the asymmetric carbon atom. CE8 can exist in many different conformations [6]. Two enantiomers $\left(E_{1}\right.$ and $E_{2}$ ) have been chosen to calculate HOF - "heat of formation". As to be expected, the energies are equal within the limits of error: $\operatorname{HOF}\left(E_{1}\right)-486.38 \mathrm{~kJ} / \mathrm{mol}$, $\operatorname{HOF}\left(E_{2}\right)-486.42 \mathrm{~kJ} / \mathrm{mol}$.

Below we present a conformation which probably is the most stable one [6]. The length of the molecule is $27.1 \AA$ and the width is $5.0 \AA[6]$.

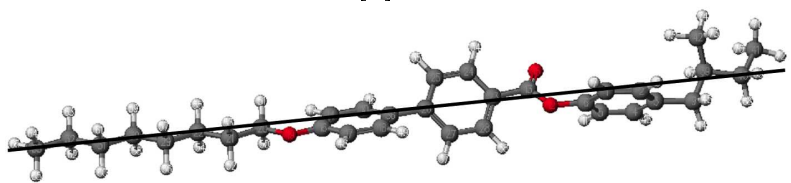

For CE8 liquid crystal we observe four smectic phases, cholesteric one and blue phase. Blue phase is stable within about 4 degrees of the temperature range. Based on texture images we have postulated the following identification of smectic phases: Sm1 mesophase (mosaic texture) as $\mathrm{SmG}$, Sm2 mesophase (fun-shaped texture) as SmI, Sm3 mesophase (fun-shaped, fingerprint texture) as $\mathrm{SmC}$ and $\mathrm{Sm} 4$ (focal conic and fun-shaped texture) as $\mathrm{SmA}$.

Finally we propose the following phase diagram:

- on heating

Cr $49.2^{\circ} \mathrm{C}$ SmG $66.5^{\circ} \mathrm{C} \mathrm{SmI} 68.9^{\circ} \mathrm{C}$

SmC $72.5^{\circ} \mathrm{C}$ SmA $139.5^{\circ} \mathrm{C}$ Ch $143.2^{\circ} \mathrm{C}$

B $149.2{ }^{\circ} \mathrm{C} \mathbf{I}$,

- on cooling

I $144.5^{\circ} \mathrm{C}$ B $141.5^{\circ} \mathrm{C}$ Ch $137.7^{\circ} \mathrm{C} \mathrm{SmA} 70.6^{\circ} \mathrm{C}$ SmC $67.8^{\circ} \mathrm{C}$ SmI $64.7^{\circ} \mathrm{C}$ SmG $43.0^{\circ} \mathrm{C}$ Cr.

Values of the molecular parameters of the investigated liquid crystal CE6.

TABLE

\begin{tabular}{c|c|c|c|c|c|c|c|c|c|c}
\hline \hline & $\begin{array}{c}\mathrm{HOF} \\
{[\mathrm{kJ} / \mathrm{mole}]}\end{array}$ & $\begin{array}{c}\mu_{x} \\
{[\mathrm{D}]}\end{array}$ & $\begin{array}{c}\mu_{y} \\
{[\mathrm{D}]}\end{array}$ & $\begin{array}{c}\mu_{z} \\
{[\mathrm{D}]}\end{array}$ & $\begin{array}{c}\mu_{\text {tot }} \\
{[\mathrm{D}]}\end{array}$ & $\begin{array}{c}\beta \\
{\left[{ }^{\circ}\right]}\end{array}$ & $\begin{array}{c}\alpha_{x} \\
{\left[10^{-41} \mathrm{~F} \mathrm{~m}^{2}\right]}\end{array}$ & $\begin{array}{c}\alpha_{y} \\
{\left[10^{-41} \mathrm{~F} \mathrm{~m}^{2}\right]}\end{array}$ & $\begin{array}{c}\alpha_{z} \\
{\left[10^{-41} \mathrm{~F} \mathrm{~m}^{2}\right]}\end{array}$ & $\begin{array}{c}\alpha \\
{\left[10^{-41} \mathrm{~F} \mathrm{~m}^{2}\right]}\end{array}$ \\
\hline$E_{1}$ & -486.38 & 2.18 & 0.50 & 0.36 & 2.27 & 164.2 & 943.0 & 447.3 & 328.1 & 572.8 \\
$E_{2}$ & -486.42 & 2.19 & 0.58 & 0.31 & 2.29 & 16.6 & 942.2 & 447.9 & 328.3 & 572.8 \\
$\mathrm{CE}^{a}$ & & & & & 2.10 & 165 & & & & 555 \\
\hline
\end{tabular}

${ }^{a}$ data presented in [6].

The calculation was performed with ChemSketch version CAChe/PM5 [6]. The total dipole moment of CE8 is almost parallel to the long molecular. It makes a relatively small angle with the long molecular axis (see Table). The long molecular axis is the axis of the lowest moment of inertia. The compound should have moderate positive dielectric anisotropy. The octyloxy chain is all-trans and in plane of the bond benzene ring. The benzene rings in the biphenyl moiety are twisted by $50.6^{\circ}$, which is in the limit of expectation compared to literature data about biphenyl. The carboxyl group - COOis twisted by a dihedral angle of $30.8^{\circ}$. More exact calculations using $a b$ initio methods yield about $0^{\circ}$ for this dihedral, which is near to experimental data in the solid state. But $a b$ initio methods cannot be applied in a large molecule like the actual one. The right benzene ring is nearly perpendicular to the middle benzene ring. The butyl chain is in all-trans conformation, and nearly perpendicular to the plane of the bond benzene ring, as usual in alkyl benzenes [6]. One may check our data comparing them with that given by Hoffmann — see Table [7].

\section{Results}

Dielectric measurements were performed at KU Leuven (Belgium) using a high-resolution dielectric analyser (Alpha Analyzer, Novocontrol Technologies) in the frequency range from $10^{-1}$ to $10^{6} \mathrm{~Hz}$. Use parallel plate capacitor resulting in thickness of the sample of $800 \mathrm{~nm}$. Subsequent dielectric measurements were done at Institute of Molecular Physics in Poznań (Poland) using HP setup in the frequency range from $10^{-2}$ to $1.5 \times 10^{6} \mathrm{~Hz}$. LINKAM $5.0 \mu \mathrm{m}$ cells was used as capacitor and this was 
calibrated. The sample was oriented by the surface effect producing a planar alignment.

Temperature dependence of $\varepsilon_{\text {stat }}$ gives a lot of information on temperature behaviour of studied substance. It is important to know how to interpret the electric permittivity value at low frequencies. Conventionally this permittivity represents the static dielectric constant. In theory it measures the response of a system to DC electric field. In real experiments it measures the response to AC low frequency electric field. For liquid crystals, frequencies below $1 \mathrm{kHz}$ could be considered as "static" ones. Finally, one can use the static dielectric diagram as a classical thermogram.

Let us analyse the plot presented in Fig. 1. Correspondence of the dielectric data to calorimetric one is distinctly visible. All smectic transition points are present. Also transition points to cholesteric phase as well as to the blue one appear. Additionally, new transition point (a "dielectric" transition) at $86^{\circ} \mathrm{C}$ occurs (for both thicknesses). As one may see going towards $\mathrm{SmC}$ phase $\varepsilon_{\text {stat }}$ increases and just after transition to the SmA drops rapidly indicating the "dielectric" transition point. Such behaviour is typical for chiral molecules where ordering effect of short range interaction is much stronger than the thermal fluctuations. "Dielectric" transition should be somehow related to this effect and will be discussed later.

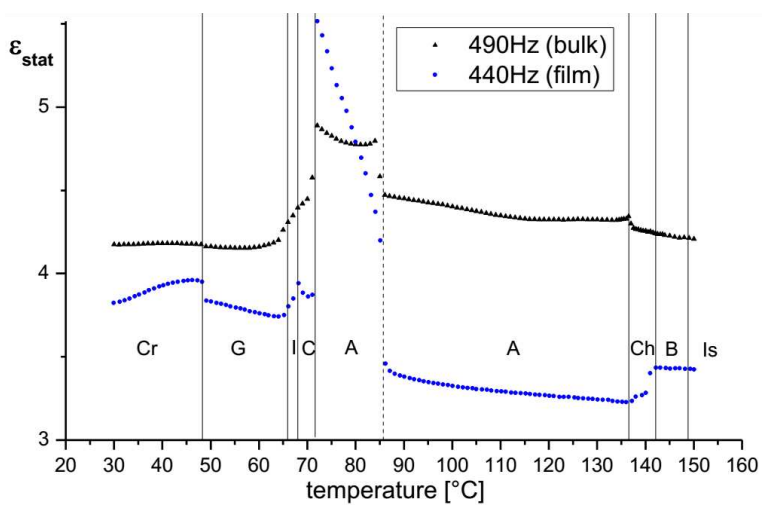

Fig. 1. Electric permittivity $\left(\varepsilon_{\text {stat }}\right)$ measured at the low ("static") frequency of $440 \mathrm{~Hz}$ (film $5.0 \mu \mathrm{m}$ ) and of $490 \mathrm{~Hz}$ (bulk $800 \mathrm{~nm}$ ). Solid lines indicate transition temperature seen via calorimetric measurement. Dash line indicates transition temperature seen in dielectric experiment. Cr means crystal state, symbols G, I, C, A stand for $\mathrm{SmG}, \mathrm{SmI}, \mathrm{SmC}$, and $\mathrm{SmA}$ phases, respectively, $\mathrm{Ch}$ is cholesteric phase and symbol B represents blue phases region, Is means isotropic state.

We want to emphasize that value of $\varepsilon_{\text {stat }}$ measured by us (for $5.0 \mu \mathrm{m}$ thick film system) is in very good agreement with data presented by Cava et al. for $25 \mu \mathrm{m}$ thick system [5]. However, one should keep in mind that they did not observe a blue phase.

Figure 2 presents the electric permittivity hysteresis loop measured at the low (static) frequency of $440 \mathrm{~Hz}$ for film $(5.0 \mu \mathrm{m})$ sample — two runs. Such behaviour was

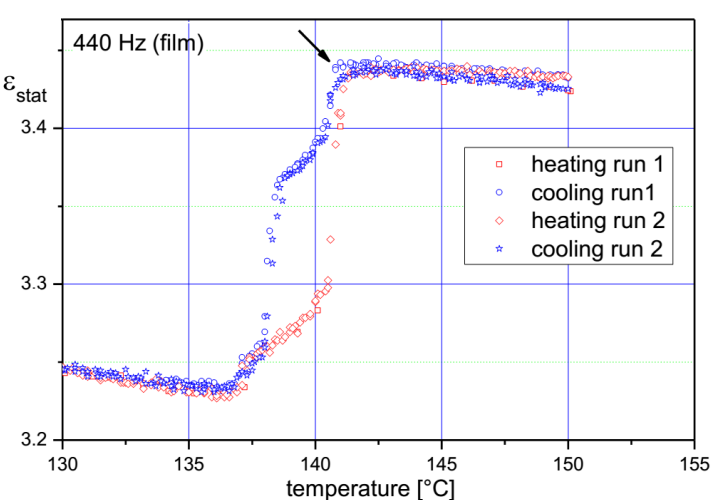

Fig. 2. Electric permittivity hysteresis loop measured at the low ("static") frequency of $440 \mathrm{~Hz}$ for film $(5.0 \mu \mathrm{m})$ sample (two runs). Arrow indicates transition temperature region to the blue phase.

observed only in the case of thin sample. In Fig. 2 one may notice the pre-transition temperature region to the blue phase. Transition to and from the cholesteric phase is clearly visible. Hysteresis loop is reproduced very well. Hysteresis occurs when the response of a physical system to an external perturbation depends on both, a magnitude of perturbation and on the previous history of the system. The chiral nematic phase exhibits ferroelectricity - nonzero spontaneous polarization (uncompensated electric dipole moments). So, being nonlinear, ferroelectric CE8 liquid crystal, investigated as the film system (5.0 $\mu \mathrm{m}$ thick), demonstrates the spontaneous polarization. Spontaneous polarization can be reversed by an applied electric field yielding the electric permittivity hysteresis loop. The chirality (noninternal plans of symmetry) generates a twisting of the molecules perpendicular to the director, with the molecular axis parallel to the director. In other words the (azimuthal) twisting from one layer to the next, produce a spiral twisting of the molecular axis which in a bulk system is compensated by volume effect.

The electric permittivity hysteresis loop may suggest that CE8 liquid crystal have ability to be easy addressing system.

The dielectric response of broad frequency range was analysed by the Havrilak-Negami (HN) equation

$$
\varepsilon^{*}(\omega)=\varepsilon_{\infty}^{\prime}+\sum_{k} \frac{\Delta \varepsilon_{k}^{\prime}}{\left[1+\left(\mathrm{i} \omega \tau_{k}\right)^{\alpha_{k}}\right]^{\beta_{k}}},
$$

where $\omega$ is the angular frequency, $\varepsilon_{\infty}$ is the high frequencies limit of the dielectric constant, $\tau_{k}$ - the mean relaxation time and $\alpha_{k}$ and $\beta_{k}$ - the shape parameters describing the symmetric and asymmetric broadening of the relaxation peak. We have to emphasis that in our case $\beta$ parameter was always one $-\mathrm{HN}$ equation was transformed into the Cole-Cole (CC) one (symmetric distribution).

Figure 3 presents the 3D plot of dielectric data ( $\varepsilon^{\prime}$ and $\varepsilon^{\prime \prime}$ ) observed on heating. However we carry out 


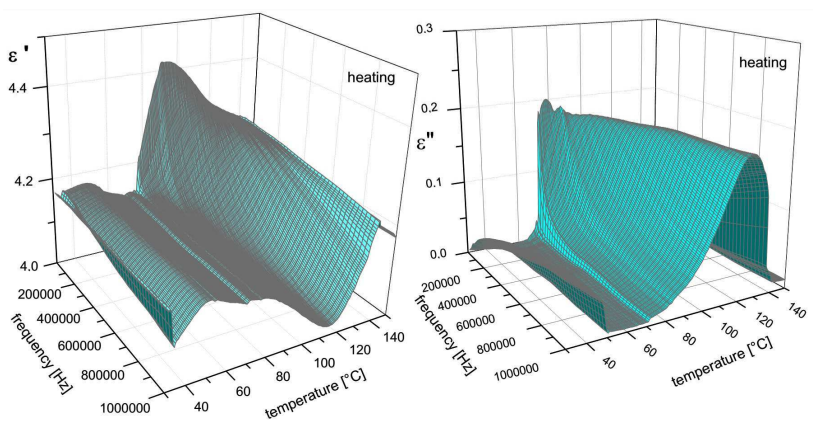

Fig. 3. The dielectric dispersion and absorption data observed on heating for CE8.

measurements on heating and cooling. We did not observe significant differences for heating and cooling runs. We noticed only some shift of transition temperatures which is common observation for liquid crystal systems. Nevertheless, for the better thermodynamic stability of the sample, the data analysis was done for cooling cycles.

The observed complex dielectric constants $\varepsilon^{*}$ were fitted to the real and the imaginary parts of $\mathrm{CC}$ equation to find the specific molecular dynamics at the different phases of investigated liquid crystal. General conclusion is that the bulk CE8 sample exhibits at least two molecular processes in each phase which for instance is clearly visible in Fig. 4 for $80^{\circ} \mathrm{C}$.

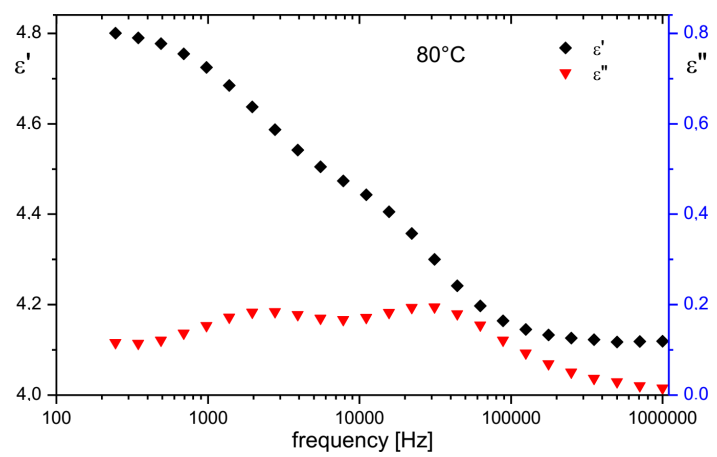

Fig. 4. The dielectric dispersion and absorption data observed at $80^{\circ} \mathrm{C}$.

Assuming a single molecular dipole relaxation as the effective response of the investigated system to an alternating field of frequency $\omega$ we decompose dielectric spectrum (observed at cooling) into sum of the ColeCole terms. At low frequencies and for high temperatures where the ohmic conduction plays an important role we evaluate conductivity $\sigma$ (Fig. 5). To extract these specific data we excluded a conductivity contribution from the complex dielectric constants $\varepsilon^{*}$. An elegant way to remove ohmic conduction from measured loss spectra uses Kramers-Kronig relation, whereby we transform the real part $\varepsilon^{\prime}$ into an imaginary part $\varepsilon_{K K}^{\prime \prime}$ that is solely based on relaxation phenomena. By virtue of this, $\varepsilon_{K K}^{\prime \prime}$ lacks the ohmic conduction term [8]. So, in turn, it indicates that resulting process is of molecular origin. Finally in the complex relaxation spectra, we can identify four modes (four processes). Fortunately for all modes $\mathrm{CC}$ parameter $\alpha$ is relatively small (varying from 0.75 to 1.00 ) (Fig. 6). Being beneficial of small $\alpha$ value it turns out that we deal with (almost) Debye-like processes. So molecular interpretation via relaxation time may be more accurate.

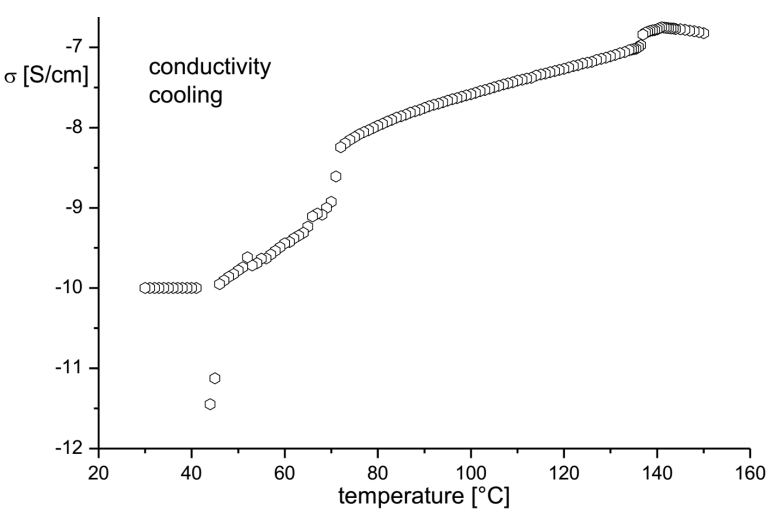

Fig. 5. The conductivity $\sigma$ calculated from complex spectra on cooling.

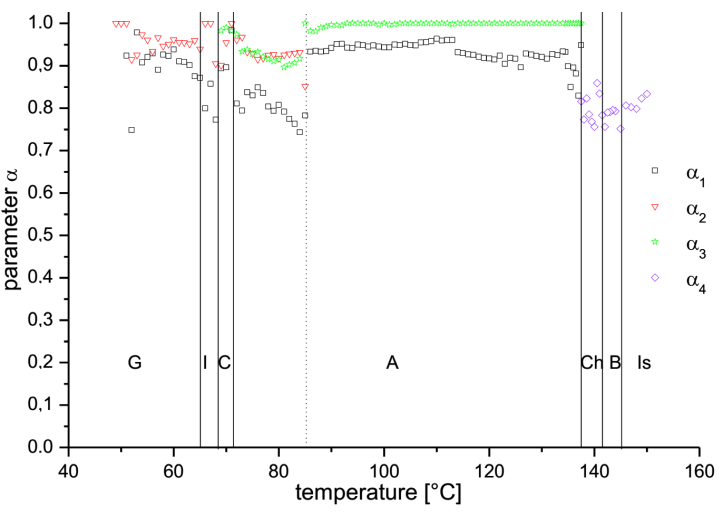

Fig. 6. The parameter $\alpha$ calculated from complex spectra on cooling. Solid lines indicate transition temperature seen via calorimetric measurement. Dash line indicates transition temperature seen in dielectric experiment. Symbols G, I, C, A stand for SmG, SmI, SmC and $\mathrm{SmA}$ phases, respectively, $\mathrm{Ch}$ is cholesteric phase and symbol $\mathrm{B}$ represents blue phases region, Is means isotropic state.

First mode, active throughout mesophases up to the transition point to cholesteric phase, is the activation process. CC parameter $\alpha$ is varying from 0.75 to 0.95 (Fig. 6). Above transition temperature to the $\mathrm{SmA}$ we deal with (almost) single time Debye-like process $(\alpha=0.95)$. The relaxation strength (dielectric amplitude) changes from 1 to maximum value 5.5 at $66^{\circ} \mathrm{C}$. Mean activation energy ( $E_{\text {a }}$ calculated from the Arrhenius plot) is $27.6 \mathrm{~kJ} / \mathrm{mol}$ in the temperature range from $47^{\circ} \mathrm{C}$ to $65^{\circ} \mathrm{C}, 24.5 \mathrm{~kJ} / \mathrm{mol}$ in the temperature range from $74^{\circ} \mathrm{C}$ to $84^{\circ} \mathrm{C}$ and $21.5 \mathrm{~kJ} / \mathrm{mol}$ in the temperature range from $95^{\circ} \mathrm{C}$ to $136^{\circ} \mathrm{C}$. 


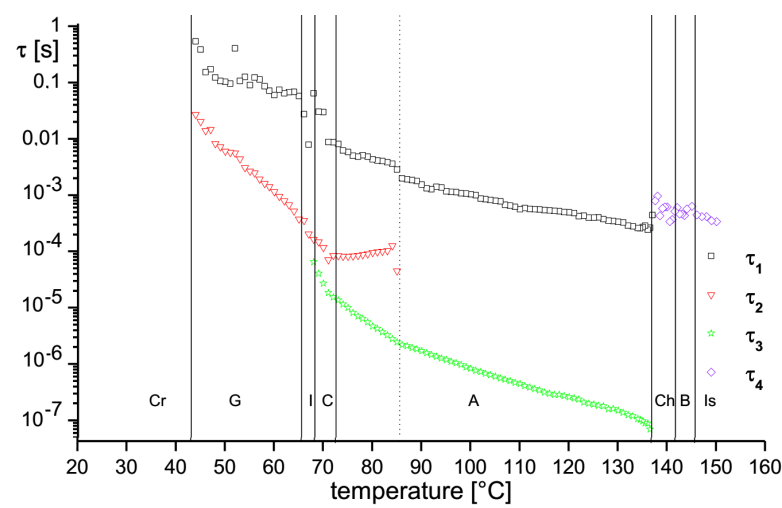

Fig. 7. The relaxation time $\tau$ calculated from complex spectra on cooling. Solid lines indicate transition temperature seen via calorimetric measurement. Dash line indicates transition temperature seen in dielectric experiment. Symbols G, I, C, A stand for SmG, SmI, SmC, and $\mathrm{SmA}$ phases, respectively, $\mathrm{Ch}$ is cholesteric phase and symbol $\mathrm{B}$ represents blue phases region, Is means isotropic state.

Second mode, active up to the "dielectric" transition point at $86^{\circ} \mathrm{C}$ phase is (almost) single time Debye-like processes; $\alpha=0.95$. In the temperature range from $47^{\circ} \mathrm{C}$ to $66^{\circ} \mathrm{C}$ activation energy is $77.5 \mathrm{~kJ} / \mathrm{mol}$ and in the temperature range from $67^{\circ} \mathrm{C}$ to $72^{\circ} \mathrm{C}$ activation energy is $115.6 \mathrm{~kJ} / \mathrm{mol}$. The relaxation strength (dielectric amplitude) is relatively small changing from 0.13 to 0.22 in the temperature range from $47^{\circ} \mathrm{C}$ to $72^{\circ} \mathrm{C}$. Above temperature $72^{\circ} \mathrm{C}$ up to $86^{\circ} \mathrm{C}$ we are dealing with nonactivation process of the relaxation strength varying from 0.89 to 1.05 .

Third mode, active throughout mesophases from $\mathrm{SmC}^{*}$ to cholesteric phase, is the activation process. In the temperature range from $65^{\circ} \mathrm{C}$ to $86^{\circ} \mathrm{C}$ ("dielectric" transition) activation energy is $73.6 \mathrm{~kJ} / \mathrm{mol}$ - process is (almost) single time Debye-like $(\alpha \propto[0.9-1.0])$. Above $86^{\circ} \mathrm{C}$ up to $138^{\circ} \mathrm{C}$ activation energy is $37.1 \mathrm{~kJ} / \mathrm{mol}$ - the process is single time Debye-like $(\alpha=1.0)$. The relaxation strength (dielectric amplitude) is relatively small $(\Delta \varepsilon=0.13)$ and temperature independent.

Fourth mode, active throughout cholesteric phase, blue phases and isotropic phase (in the vicinity of transition point) is the activation process. The activation energy is $27.6 \mathrm{~kJ} / \mathrm{mol}$. Process is Cole-Cole type $(\langle\alpha\rangle=0.8)$; one may expect distribution of the relaxation time. The relaxation strength (dielectric amplitude) is relatively small $(\Delta \varepsilon \approx 0.1$ ) and temperature independent.

\section{Molecular interpretation}

It is shown that almost all observed processes are characterized by $\alpha$ parameter practically equal to 1 - the Debye processes (Fig. 8). Only first process for some mesophases seems to be complex one $-\alpha$ parameter changes from 0.75 to 0.95 . The effect of the $\alpha$ parameter is commonly interpreted as a measure of a symmetric distribution of the dielectric relaxation times. In the real systems, local fluctuations of molecular structure or environment (the long-range dipolar correlation) which is responsible for a "dielectric friction" might result in a distribution of relaxation times. However, we should note that the translational motion may reduce distribution of relaxation times [4]. So, one might expect Debye like relaxation in liquid crystals.

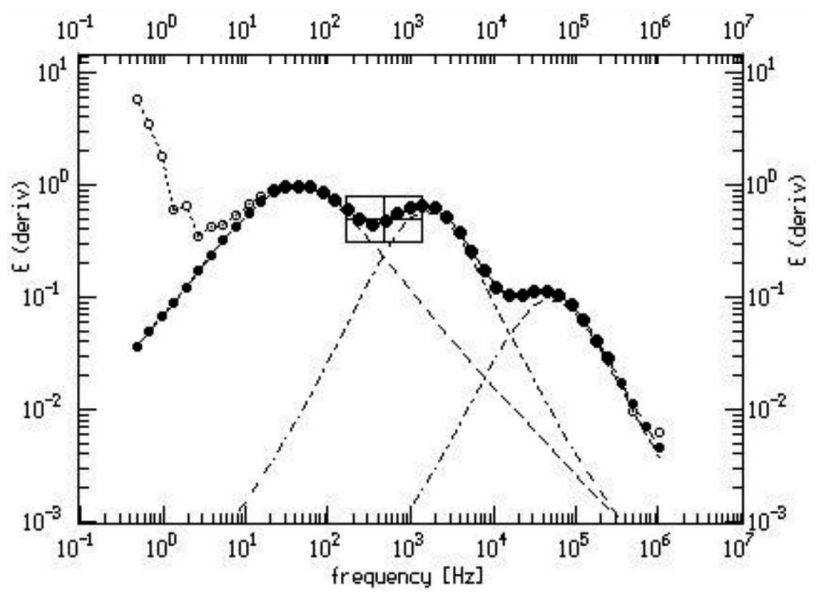

Fig. 8. Example of the complex spectra decomposition graph at $83^{\circ} \mathrm{C}$. Ordinate axis shows the derivative of absorption $\left(\Delta \varepsilon_{1}=2.25, \alpha_{1}=0.76, f_{1}=41.9 \mathrm{~Hz}, \Delta \varepsilon_{2}=\right.$ $0.89, \alpha_{2}=0.93, f_{2}=1.55 \mathrm{kHz}, \Delta \varepsilon_{3}=0.89, \alpha_{3}=0.91$, $\left.f_{3}=48.9 \mathrm{kHz}\right)$.

Nordio-Rigatti-Segre (NRS) gave the theory for the relaxation of the complex dielectric permittivity in the local anisotropic potential of the liquid crystal state [9]. NRS theory relates the complex permittivity $\varepsilon^{*}$ to the dipole moment components $\mu_{\mathrm{l}}$ and $\mu_{\mathrm{t}}$ of the mesogen. Because the effective dipole moment is normalized with respect to the free-molecule dipole moment, it is clear that finally the authors arrive at four different relaxation $\operatorname{modes}_{\mathrm{NMR}}\left(M_{p q}, p, q=0,1\right)$ : two for homeotropic orientation $\left(\mathrm{M}_{00}\right.$ and $\left.\mathrm{M}_{01}\right)$ and two for planar one $\left(\mathrm{M}_{10}\right.$ and $\mathrm{M}_{11}$ ) [4]. Each components of the effective dipole moment contains two contributions from the molecular dipole moment, and each can have different relaxation time. However for a uniaxial molecule with two independent moments of inertia, the dynamics can be approximately described in terms of three different relaxation times (or frequencies) [4]. Ferrarini et al. [10] have evaluated numerically NRS theory. They have confirmed the existence of three different relaxation time modes. First one results from end-over-end rotation. This mode is strongly dependent upon the order parameter (in other words this mode is strongly temperature dependent). It is characterized by a long time scale. Second one is rotation about its own long molecular axis. It is characterized by an average time scale. Third one is precessional motion about director. It is characterized by a very short time scale.

Let us begin the molecular description of four modes observed by us from mode one:

- First mode has relatively big dielectric amplitude (one order of magnitude higher than the others). 
Its activation energy decreases slightly with increasing temperature (from $27.6 \mathrm{~kJ} / \mathrm{mol}$ at $47^{\circ} \mathrm{C}$ to $21.5 \mathrm{~kJ} / \mathrm{mol}$ at $136^{\circ} \mathrm{C}$ ). Because the total dipole $\left(\mu_{\text {tot }}=2.27 \mathrm{D}\right)$ is almost parallel to the long molecular $\left(\mu_{x}=2.18 \mathrm{D}\right)$ axis and dielectric spectrum is proportional to the dipole moment we suggest that molecular origin of this process is the reorientation around the short molecular axis (end-over-end rotation). Process is visible over all smectic phases which may support our interpretation. Process is slow - at transition temperature to $\mathrm{SmG}$ phase relaxation time is even of the order of seconds - which might be due to the dense molecular packing seen for instance in X-ray experiment [11]. This is consistent with the above description (NMR theory). Ferroelectric liquid crystal (D)-2-(2-bromo-3-methyl-butanoyloxy)-6-(4- $n$ -decyloxybenzoyloxy)-naphthalene $(\mathrm{Br} 4)$ (which is almost rod-like molecule - the length $36.5 \AA$ and the width is $8.5 \AA$ ) has the total dipole $\left(\mu_{\text {tot }}=\right.$ $3.3 \mathrm{D})$ almost parallel to the long molecular $\left(\mu_{1}=\right.$ $2.7 \mathrm{D})$. For this substance we have observed similar process. We assigned it to the reorientation around the short molecular axis [12].

- Second mode, visible throughout "crystal-like" mesophases and partially in SmA phase (up to $86^{\circ} \mathrm{C}$ ) is the Debye processes $(\alpha \approx 0.95)$. This mode is the activation one throughout "crystal-like" mesophases (from $47^{\circ} \mathrm{C}$ to $66^{\circ} \mathrm{C}, E_{\mathrm{a}}=77.5 \mathrm{~kJ} / \mathrm{mol}$ and from $67^{\circ} \mathrm{C}$ to $72^{\circ} \mathrm{C}, E_{\mathrm{a}}=115.6 \mathrm{~kJ} / \mathrm{mol}$ ). Molecular origin of this process is the reorientation of the rigid core about long molecular axis. Taking into account that relatively high value of the activation energy increases with increase of temperature we suggest a dipole-dipole correlation effect resulting in the dielectric friction. Electric permittivity $\left(\varepsilon_{\text {stat }}\right)$ decreases going towards transition point to SmI phase for both thin and thick samples (see Fig. 1). So, we suggest formation of dimers (molecular pairs) giving as a result antiparallel order in the short range. Going towards transition point to SmA phase we observe increase of electric permittivity $\left(\varepsilon_{\text {stat }}\right)$ (especially prominent for thin sample). Therefore, we suggest formation of dimers (molecular pairs) giving as a result parallel order in the short range. For parallel ordering we may expect strong positive dipole correlation and in turn higher energy needed to perform reorientation. Certain way to describe how strong may be this effect is to introduce the dipole-dipole correlations $g$-factor. Because of lack of some molecular data we cannot calculate $g$-factor for the molecule under investigation. However, one may take into consideration $g$-factor for similar substance namely (4'-hexyloxybenzoate) 4-(2-methylobuthyl) phenol (CE6).

Figure 9 presents temperature dependence of anisotropic dipole-dipole correlation factor $g$ for

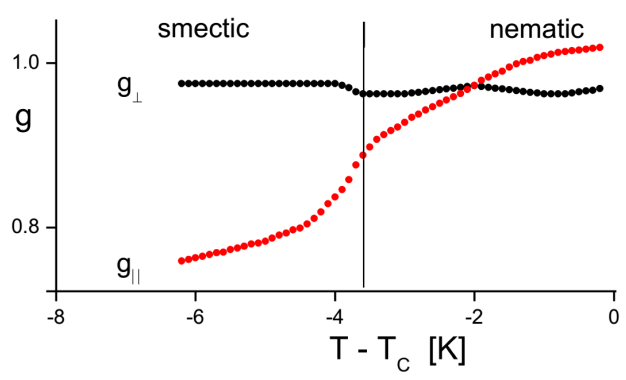

Fig. 9. Temperature dependence of $g$-factor obtained for CE6 for the smectic and nematic phases.

parallel and perpendicular components of the dipole moment of CE6 in smectic and nematic phases [13]. As it is seen for the same substance, correlation as a function of temperature may change from antiparallel towards parallel. These conclusions are in agreement with calculated activation energy values. Within SmG phase where we suggest antiparallel order the activation energy is smaller than for $\mathrm{SmI}$ and $\mathrm{SmC}$ phases where we suggest parallel order. At transition $\mathrm{SmC}-\mathrm{SmA}$ point we observe sudden increase of the electric permittivity $\left(\varepsilon_{\text {stat }}\right)$. On the other hand, above $\mathrm{SmA}$ transition it is discovered that the reorientation under discussion is not thermally activated process till the dielectric transition point. We think that strong positive dipole correlation forms dimers (molecular pairs) of ferroelectric order in the short range. Finally, for this reason, molecularly we interpret this process as a linear response of statistically oriented ferroelectric clusters. Increasing temperature and hence increasing thermal energy tries to destroy dimers structure and slows down cluster reorientation. Finally, above $86^{\circ} \mathrm{C}$ process (dimers structure) disappears. We may say that a small break seen on time $\left(\tau_{3}\right)$ vs. temperature curve for third mode at $86^{\circ} \mathrm{C}$ ("dielectric" transition) is related to disappearance of dimers structure, too. Let us note that this description is consistent with the NRS theory.

- Third mode, visible throughout "liquid-like" mesophases is the fasted one. We assume that the foregoing Debye process may be due the conformational motion (intra reorientation) or precessional motion about director of the CE8 molecule. $\mathrm{Up}$ to $86^{\circ} \mathrm{C}$ process has comparatively high activation energy (about $70 \mathrm{~kJ} / \mathrm{mol}$ ). This confirms the existence of dipole-dipole interaction. Above $86^{\circ} \mathrm{C}$ the activation energy drops down (up to about $40 \mathrm{~kJ} / \mathrm{mol}$ ) reaffirming disappearance of dipole-dipole interaction. The activation energy of about $40 \mathrm{~kJ} / \mathrm{mol}$ is proper for this reorientation observed for instance for 1,3-phenylene bis[4-(10-undecyloxy)-benzoiloxy] benzoates [14]. We know from calculations for the isolated molecule (using 
the quantum chemical program $\mathrm{MOPAC} / 7$, with the AM1 Hamiltonian [11]) that the CE8 molecule can exist in many different conformations. So, conformational changes as the molecular interpretation of third mode are likely. Precessional motion is also possible and is in accordance with the NRS theory.

- Fourth mode of relatively small relaxation strength (dielectric amplitude) is the Cole-Cole type throughout cholesteric phase, blue phases and isotropic phase. This mode seems to be superposition of processes. Within the cholesteric phase as well as blue phases we may assume that molecular origin of foregoing process is reorientation of the transverse dipole moment components. The freedom reorientations around the long molecular in the case of twisted structure and bulk sample is restricted. Thus, the activation energy of $27.6 \mathrm{~kJ} / \mathrm{mol}$ is reasonable (for instance for $7 . \mathrm{OAOB}$ in nematic phase $E_{\text {long }}=24 \mathrm{~kJ} / \mathrm{mol}$ as presented in [15]). In the blue phases where the regular three-dimensional cubic structure appears the activation energy is unchanged. For "bulk" measurements slowing-down effect is similar within spatial structure of cholesteric and blue phases. According to the Nordio-Rigatti-Segre theory, in the isotropic phase, one might observe two main processes: reorientations around the long and short molecular axes (so we observe Cole-Cole process). However, in the isotropic phase long molecular axes are distributed almost randomly. So, reorientation around the long molecular axis which is mainly responsible for this mode may be assumed to be as restricted as for cholesteric and blue phases. We do not observe any change in the activation energy at transition point. To compare the activation energy of $7 . \mathrm{OAOB}$ in isotropic phase is $E_{\text {iso }}=27 \mathrm{~kJ} / \mathrm{mol}$ (as presented in [15]). The relaxation strength (dielectric amplitude) which is temperature independent throughout discussed phases also confirms molecular interpretation of mode fourth. Intentionally we did not apply to CE8 sample any external ordering agent. So, symmetric distribution of relaxation time (the Cole-Cole process) is a natural consequence of "bulk" dielectric measurements. This process is relatively slow (the relaxation time $\tau$ of the order of ms). As it was suggested by Hoffmann, the CE8 liquid crystal in "static" conditions will act as an isotropic dielectric [7]. Thus our statement that we are dealing with symmetric distribution of relaxation time is legitimate.

\section{Conclusions}

For (4-(4'octylobiphenyl)carboxylan) 4-(2-methylobuthyl) phenol we observe complex molecular dynamic in all mesophases including blue phase. Throughout smectic phases we identify at least two processes. The analysis of the dielectric relaxation processes allows to make a general statement; we deal with Debye-like or almost
Debye-like molecular processes. The origin of observed processes is reorientation of the rigid core about the short and long molecular axes as well as intra reorientation. We noticed effect of dipole-dipole interaction seen via ferroelectric cluster reorientation. In the cholesteric, blue and isotropic phases we classified the relaxation processes as the Cole-Cole type. The molecular origin of studied processes is mainly reorientation of the transverse dipole moment components due to rotation around the long molecular axis.

\section{Acknowledgments}

The authors would like to thank specially Profs. W. Kuczyński and J. Hoffmann as well as Prof. Michael Wübbenhorst and Bram Vanroy for help in measurements and valuable discussions.

\section{References}

[1] H.J. Coles, M.N. Pivnenko, Nature 436, 997 (2005).

[2] J. Yamamoto, I. Nishiyama, M. Inoue, H. Yokoyama, Nature 437, 525 (2005).

[3] S. Meiboom, J.P. Sethna, W.P. Anderson, W.F. Brinkman, Phys. Rev. Lett. 46, 1216 (1981).

[4] W. Otowski, in: Molecular motion in liquid crystals as seen via dielectric spectroscopy, in: Dielectric Properties of Liquid Crystals, Eds.: Z. Galewski, L. Sobczyk, Transworld Research Network, 2007, p. 1.

[5] R.J. Cava, J.S. Patel, E.A. Rietman, J. Appl. Phys. 60, 3093 (1986).

[6] D. Demus, private communication.

[7] J. Hoffmann, Acta Phys. Pol. A 105, 373 (2004).

[8] M. Wübbenhorst, J. van Turnhout, J. Non-Cryst. Solids 305, 40 (2002).

[9] P.L. Nordio, G. Rigatti, U. Segre, Mol. Phys. 25, 129 (1973).

[10] A. Ferrarini, P.L. Nordio, G.J. Moro, in: The Molecular Dynamics of Liquid Crystals, Eds. G.R. Luckhurst, C.A. Veracini, NATO ASI Series, Series C, 1994, p. 41.

[11] G. Lewińska, W. Otowski, Acta Phys. Pol. A 117, $562(2010)$.

[12] W. Otowski, Y. González, B. Palacios, M.R. de la Fuente, M.A. Pérez Jubindo, Ferroelectrics 180, 93 (1996).

[13] W. Otowski, W. Demol, W. van Dael, Mol. Cryst. Liq. Cryst. 226, 103 (1993).

[14] W. Otowski, A. Budziak, A. Dobrowolska, Mol. Cryst. Liq. Cryst. 494, 1 (2008).

[15] X.P. Nguyen, W. Otowski, S. Wróbel, S. Urban, J. Chruściel, H. Kresse, Z. Naturforsch. 40a, 627 (1985). 